Recepción: 31 / 08 / 2016

Aceptación: 25 / 01 / 2017

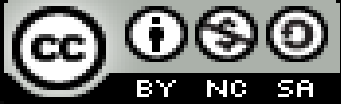

Ciencias sociales y políticas

Publicación: 29 / 04 / 2017

Artículo de investigación

\title{
La resiliencia y su impacto por el terremoto del 2016 en Manta, Ecuador
}

\author{
Resilience and its impact from the 2016 earthquake in Manta, Ecuador \\ Resiliência e seu impacto pelo terramoto de 2016 em Manta, Equador
}

\author{
Patrícia J. López-Mero \\ pattylopez@hotmail.com \\ María C. Pibaque-Tigua ${ }^{\mathrm{II}}$ \\ mary35bebe@hotmail.com
}

Richard F. Rodríguez-Andrade ${ }^{\mathrm{II}}$ richard.rodriguez@uleam.edu.ec

Cinthya E. Merchán Briones ${ }^{\mathrm{IV}}$

ce.merchan@gmail.com

\section{Correspondencia: pattylopez@ hotmail.com}

\footnotetext{
${ }^{\mathrm{I}}$ Magister en Gerencia Educativa, Diplomado Superior en Intervención Social, Licenciada en Ciencias de la Educación, Licenciada en Trabajo Social Especialidad Atención a la Familia y al Desarrollo Comunitario, Docente de Segunda Enseñanza Especialidad Castellano y Literatura, Docente de la Universidad Laica Eloy Alfaro de Manabí, Manta Ecuador.

${ }^{\text {II }}$ Magister en Educación Parvularia, Licenciada en Trabajo Social, Docente de la Universidad Laica Eloy Alfaro de Manabí, Manta Ecuador.

III Magister en Gerencia Educativa, Analista de Sistemas, Licenciado en Ciencias de la Educación, Docente de Educación Pre-Primaria Nivel Técnico Superior, Docente de la Universidad Laica Eloy Alfaro de Manabí, Manta Ecuador

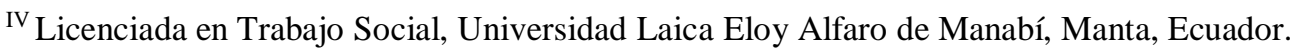




\title{
Resumen
}

Se realizó una investigación cuantitativa, cualitativa, descriptiva y transversal, en la parroquia Tarqui del cantón Manta, con vista a determinar la influencia de la resiliencia en el desarrollo social de las personas afectadas por el terremoto del 16 de abril 2016. En la serie se obtuvo que mantener una actitud positiva es una de las características de la resiliencia que ayuda en el desarrollo social, porque de esta manera pueden continuar con el desarrollo vivencial, permitiendo ver los momentos desesperantes, pero que la vida continúa y se debe salir adelante, se logró conocer varios factores que se mantuvieron en el aprestamiento de los acontecimientos, causas como la pérdida del casco comercial, familiares e infraestructura; se logró identificar la importancia que tiene la intervención del trabajador social según sus funciones, por otra parte, lleva a cabo una adaptación positiva a pesar de las importantes agresiones sobre el proceso de desarrollo, donde interviene de forma directa el desarrollo social como un cambio en el comportamiento de una sociedad, de una comunidad.

Palabras clave: resiliencia; desarrollo social; trabajo social; desastres naturales.

\begin{abstract}
A quantitative, qualitative, descriptive and cross-sectional investigation was carried out in the Tarqui parish of Manta canton with a view to determining the influence of resilience on the social development of the people affected by the earthquake of April 16, 2016. The series was obtained that maintaining a positive attitude is one of the characteristics of the resilience that helps in the social development, because in this way they can continue with the experiential development, allowing to see the desperate moments, but that the life continues and one must succeed, was achieved to know several factors that remained in the preparation of the events, causes like the loss of the commercial helmet, familiar and infrastructure; it was possible to identify the importance of the intervention of the social worker according to his functions, on the other hand, he carries out a positive adaptation in spite of the important aggressions on the development process, where social development is directly involved as a change in the behavior of a society, of a community.
\end{abstract}

Keywords: resilience; social development; social work; natural disasters. 


\section{Resumo}

Uma pesquisa quantitativa, qualitativa, descritiva e transversal que realizei na freguesia Tarqui da Manta cantão com vista para determinar a influência de resiliência no desenvolvimento social das pessoas afetadas pelo terremoto em 16 de Abril de 2016. Foi obtida a série para manter uma atitude positiva é uma das características de resiliência que ajuda o desenvolvimento social, pois desta forma pode continuar o desenvolvimento experimental, permitindo que você veja os momentos de desespero, mas a vida continua e deve avançar, foi possível saber vários fatores que permaneceram na preparação de eventos, causas e perda de centro comercial, família e infraestrutura; Foi possível identificar a importância da intervenção do trabalho social de acordo com as suas funções, por outro lado, ele executa uma adaptação positiva apesar assaltos significativos no processo de desenvolvimento, que intervém desenvolvimento directamente social como uma mudança na o comportamento de uma sociedade, uma comunidade.

Palavras chave: resiliência; desenvolvimento social; trabalho social; desastres naturais.

\section{Introducción}

La resiliencia se refiere a «un proceso dinámico que abarca la adaptación positiva dentro del contexto de una adversidad significativa" (Fernández, 2004, p.43). Esto implica que el sujeto, por una parte, va a ser expuesto a una amenaza significativa o a una adversidad severa y, por otra parte, lleva a cabo una adaptación positiva a pesar de las importantes agresiones sobre el proceso de desarrollo.

Significa una combinación de factores que permiten al ser humano, afrontar y superar los problemas y adversidades de la vida, y construir sobre ellos. La resiliencia distingue dos componentes: la resistencia frente a la destrucción; es decir, la capacidad de proteger la propia integridad bajo presión; por otra parte, más allá de la resistencia, es la capacidad de forjar un comportamiento vital positivo pese a circunstancias difíciles.

Lagos P. (2016) mantiene que la idea de resiliencia como algo absoluto y global, ha cambiado en los últimos años, pasando a ser considerada en este momento como algo relativo, que depende del equilibrio dinámico de factores personales, familiares, sociales y también de los momentos del 
ciclo vital; y especifico, generalmente circunscrito a determinadas áreas de adaptación psicológica.

Distintos investigadores conceptúan la resiliencia como la suma de factores individuales, familiares y sociales, así como una función de factores de protección compuestos de recursos personales y sociales. La base de la resiliencia también ha sido descrita como consistente de atributos diposicionales, uniones familiares afectivas y apoyo externo.

La resiliencia resulta importante, en tanto a partir de su conocimiento es posible diseñar políticas de intervención. A partir de esto, la intervención desde un punto de vista clínico puede ser concebida como un intento de alterar el balance presente en las personas, que oscila desde la vulnerabilidad a la resiliencia. (Melillo, Suárez Ojeda, 2002, p.83).

Esto puede ocurrir ya sea, disminuyendo la exposición a situaciones de vida provocadoras de estrés y que atentan contra la salud mental, o bien aumentando o reforzando el número de factores protectores que pueden estar presentes en una situación dada.

Cabe agregar que la resiliencia se ha caracterizado como un conjunto de procesos sociales e intrapsíquicos que posibilitan tener una vida "sana" en un medio insano. Estos procesos se realizan a través del tiempo, dando afortunadas combinaciones entre los atributos del ser humano y su ambiente familiar, social y cultural. (Suárez, 2003, p.104).

Así la resiliencia no puede ser pensada como un atributo con que las personas nacen o adquieren durante su desarrollo, sino que se trata de un proceso que caracteriza un complejo sistema social, en un momento determinado del tiempo.

Las personas no reaccionan de la misma forma a los mismos eventos de vida traumáticos y estresantes. "Un enfoque para construir resiliencia que le funciona a una persona puede no funcionarle a otra“(Grotberg, 2002, p.22). Las personas utilizan una gran variedad de estrategias.

\section{Algunas variaciones pueden reflejar diferencias culturales}

Reconocer la propia fortaleza y recursos para tratar con situaciones difíciles puede ayudar a desarrollar confianza en sí mismo. Prestar atención a sus necesidades y sentimientos, otras formas 
de fortalecer la resiliencia pueden ayudar, la clave es identificar formas que podrían resultar bien como parte de una estrategia personal para desarrollar resiliencia. (Kinard, 2008, p.35).

Trabajar en el campo de lo resiliente en un individuo implica un cambio paradigmático en el sentido de hacer hincapié en la fortaleza innata y ver las cosas como algo positivo y no como riesgo total, las cosas se han creado para mejorar su entorno no para dañar su salud.

Una adecuada estimulación en los primeros años de vida tendrá un gran beneficio para el futuro puesto que el apoyo familiar y la integración hacen que una persona desarrolle confianza en sí mismo y esto en un futuro se verá reflejado en los proyectos y el éxito o fracaso que se obtenga de éstos. (Sánchez, 2003).

La resiliencia puede ser promovida, por lo tanto es necesario estudiar la elaboración de modelos que permitan promoverla de forma efectiva mediante programas de intervención". (Gómez, 2010).

Por las consideraciones anteriores la resiliencia nos indica, la necesidad de focalizar nuestra búsqueda en los recursos personales y ambientales que disponen los individuos, sus familias y la comunidad. Y se cambia, desde una intervención en el beneficiario directo a una intervención que incorpora a la familia y a la comunidad durante todo el proceso de cambio. Se incorporan actividades educativas que abordan las distintas dimensiones de la resiliencia. (García \& Soria, 2010, p.11).

Se deben impulsar acciones para favorecer a la inclusión social de la resiliencia, para que se aproveche la vitalidad, la capacidad y la energía para participar activamente en el presente y construir con el apoyo un proyecto de vida, tenemos que considerar acciones y características que promuevan la resiliencia y este tema debiese ser prioritario.

Promover la resiliencia es reconocer la fortaleza más allá de la vulnerabilidad. Apunta a mejorar la calidad de vida de las personas a partir de sus propios significados, según ellos perciben y se enfrentan al mundo. Nuestra primera tarea es reconocer aquellos espacios, cualidades y fortalezas que han permitido a las personas enfrentar positivamente experiencias estresantes. Estimular una 
actitud resiliente implica potenciar esos atributos incluyendo a todos los miembros de la comunidad en el desarrollo, la aplicación y la evaluación de los programas de acción.

La práctica del trabajo social en el siglo XXI requiere nuevos enfoque teóricos que permitan a los trabajadores sociales basarse en las capacidades de los clientes para persistir frente a los obstáculos y proceder positivamente con los acontecimientos de la vida. (Forés, 2008).

Los trabajadores sociales están comprometidos desde hace tiempo con prácticas basadas en las fortalezas, capacidades y protecciones de los usuarios, éstos son los factores que le movilizan, motivan y orientan hacia su crecimiento, su autocuidado y autodirección.

Otro eje de intervención desde el trabajo social es la activación de la resiliencia en las familias que atraviesan un conflicto o un problema.

El secreto está en ayudar a las familias a fortalecerse, a reconocer sus fortalezas y confiar en ellas, y a adquirir mayor conciencia social para promover cambios que reduzcan la inequidad y el sufrimiento. No existe un modelo de familia, ni de madre, ni de hijos, sino familias, madres, padres e hijos que se relacionan de modos diversos, y esas formas de relación están estrechamente ligadas a sus historias personales de vida y de ver el mundo, que a menudo no es igual a la nuestra.

Se puede hablar de desarrollo social en cualquier contexto histórico y geopolítico, específicamente en relación con el mejoramiento de las condiciones de vida de una sociedad, los cambios sociales suceden gracias al aporte de los llamados pioneros, personajes que primero experimentan el cambio y lo trasladan después a la sociedad a la que pertenecen, con el firme propósito de buscar su modificación. (Elementos para lograr un desarrollo social integral. 2011).

A toda persona de bien le preocupa el progreso y el desarrollo social, de su país, de su tierra, de su nación, Es por esta razón que el nivel de desarrollo aumenta de manera favorable a como aumentan la organización de la sociedad y sus niveles de conocimiento. 
Es la socialización del proceso por el cual cada ser humano se convierte en un miembro activo y de pleno derecho de la sociedad de la que forma parte. Haciendo énfasis en una variación vertical del nivel cualitativo de las actividades, (Martínez A. 2007).

Estas son las nuevas ideas, las prácticas y hábitos, en principio son obstaculizados por los elementos conservadores de la sociedad, pero, en ocasiones, si son funcionales y en realidad son claramente un dinamizador de un desarrollo social efectivo, son aceptados, imitados y organizados, con una mayor fruición de parte de la sociedad en la cual se efectúan, sin embargo, no hay un acuerdo unánime para determinar o dar énfasis a una variación vertical del nivel cualitativo de las actividades.

Con la actual investigación se pretende exponer los aspectos que se manifiestan en cuanto a la resiliencia como acto de anteponerse a cualquier suceso, en este caso por todos los aspectos con enfoque sociales en el desarrollo de las personas afectadas por el terremoto ocurrido el 16 de Abril 2016.

\section{Metodología}

Se realizó una investigación cuantitativa, cualitativa, descriptiva y transversal en la parroquia Tarqui del cantón Manta con el fin de determinar la influencia de la resiliencia en el desarrollo social de las personas afectadas por el terremoto del 16 de abril 2016.

El estudio fue aplicado en la parroquia Tarqui, donde es extensamente el daño más grande, ahora denominada zona cero, donde más de 4 mil familias, vendedores formales e informales tuvieron pérdidas no solo materiales sino humanas, en donde se brindó una orientación y atención directa a la problemática dando respuesta mediante acciones sociales que determinó las vivencias familiares y la carencia de la atención al desarrollo social que deben ejercer este grupo de personas, conociendo también que existen cerca de 1800 personas que oscilan en problemas vivenciales, tendencias a no poder controlar las emociones ante las réplicas que se generaron, temor al dormir pensando en el bienestar personal o familiar, en los actuales momentos creció la taza en personas en cuanto a la fobia de permanecer en edificios altos, o estar en lugares donde consideran ellos se pueda producir un derrumbamiento. 
Para el cumplimiento al objetivo planteado en un proceso de investigación, cualquiera sea su naturaleza, requiere la selección y aplicación de métodos, técnicas y procedimientos que orienten el camino a su consecución. Es por esta razón que para el desarrollo del trabajo de investigación propuesto se utilizó los siguientes métodos:

Método inductivo: Este método se utilizó en el momento de inducir el requerimiento investigativo a los dirigentes encargados en el desarrollo sustentable en el denominado ahora “Zona cero" (personas de la parroquia Tarqui) que fueron objeto de estudio.

Método deductivo: Este método se empleó para concluir la información obtenida de los actores de la indagación.

Método analítico sintético: Se estudió y revisó todos los datos compilados en las encuestas, observación y entrevistas que se aplicaron a la población.

De acuerdo a la segmentación poblacional determinado, el área geográfica es el sector en que se desarrolló la investigación, la misma cuenta con una población económicamente activa de aproximadamente 89.025 habitantes. (INEC 2010).

Se utilizó el tipo de muestreo probabilístico aleatorio simple, el cual se toma elementos poblacionales utilizando un procedimiento completamente aleatorio; 383 habitantes afectados por el evento natural. Permitiendo llegar de forma más representativa al mercado objetivo como es el caso de las personas afectadas por el terremoto.

Asimismo en el estudio se describieron las categorías fundamentales relacionadas con la resiliencia a través de la categoría: importancia, características y clasificación, con respecto al desarrollo social las categorías que se tuvieron en cuenta fueron: importancia del desarrollo social, factores del desarrollo social, fases del desarrollo social, ajuste en el desarrollo social.

Las técnicas usadas fueron:

La entrevista: este instrumento se utilizó para obtener información de parte de las profesionales encargados de los sucesos dados en la zona cero Parroquia Tarqui. 
Encuesta: este instrumento se empleó con un banco de preguntas a las personas afectadas en el terremoto en la parroquia Tarqui.

\section{Análisis y resultados}

Resultado de la entrevista aplicada a profesionales participes en la ayuda del terremoto del 16 de abril en la zona Tarqui en la ciudad de Manta.

Al realizar la entrevista a los profesionales que participaron en la ayuda del terremoto, en la pregunta sobre cuál es su profesión y la función que ejerció en el evento natural, intervinieron un abogado, trabajador social, ingeniero, su labor fue como voluntario ayudando a las personas afectadas y colaborando con el Departamento de Gestión y Riesgo.

En cuanto al conocimiento que poseían acerca de la resiliencia expresaron tener poco conocimiento.

Respecto a si la resiliencia influye significativamente en el desarrollo social de las personas afectadas por el terremoto, se expresaron positivamente, ya que depende del grado de desarrollo que las personas afectadas tengan respecto a la resiliencia.

Sobre las actividades que se realizaron ante el suceso del 16 de abril, los entrevistados plantearon que, las personas más afectadas por el terremoto en la zona cero fueron quienes perdieron a sus familiares, quienes perdieron sus viviendas y sus negocios.

Al desarrollar la entrevista a los profesionales que laboraron en el terremoto, con respecto a cuáles son las características de la resiliencia que intervienen en el desarrollo social de las personas afectadas, consideraron que el positivismo ante las adversidades es una característica que ha sobresalido mucho en las personas que han sido afectadas por el terremoto, también el deseo de superación de no quedarse en el papel de víctimas esperando recibir las ayudas que llegan sino que se desenvuelven por ellos mismos ante esta situación que se les presentó en sus vidas.

Al indagar sobre cuáles serían las estrategias de la resiliencia que fortalecen el desarrollo social de las personas afectadas el abogado expresó que una de las estrategias sería forjarse metas, 
determinar lo que quieren alcanzar y establecer de qué manera lo van a conseguir, es muy importante que se direccionen y trabajen por ello, otra de las estrategias planteadas por el trabajador social y el ingeniero fue asignarse metas a corto y largo plazo en lo que respecta a lo laboral, hacer planes, establecer objetivos y dar el paso, es importante que tengan claros cuáles son sus objetivos y que actúen para alcanzarlos.

Resultados de la encuesta aplicada a las personas afectadas por el terremoto de la zona de Tarqui en el cantón Manta

El 50\% de las personas encuestadas se encontraban en un rango de edad entre 36 - 50 años, mientras que un $36 \%$ entre 21 - 35 años, el $11 \%$ de 51 años en adelante y un $3 \%$ se encontraban en un rango de edad entre $14-20$ años.

Tabla 1. ¿Qué tipo de ayuda recibió después del terremoto?

\begin{tabular}{|l|l|l|l|}
\hline $\mathbf{N}^{\mathbf{0}}$ & Alternativas & Frecuencia & Porcentaje \\
\hline a) & Psicológica & 109 & $29 \%$ \\
\hline b) & Social & 124 & $32 \%$ \\
\hline c) & Policial & 150 & $39 \%$ \\
\hline & & 383 & $100 \%$ \\
& Total & & \\
\hline
\end{tabular}

En la serie prevalecieron las personas que manifestaron que el tipo de ayuda que recibieron después del terremoto fue policial (39\%), 32 \% dijeron que el tipo de ayuda que recibieron después del terremoto fue social y un $29 \%$ expresaron que el tipo de ayuda que recibieron después del terremoto fue psicológica, como se observa en la tabla 1. 
Tabla 2. Puede tener la fortaleza y estar preparado/a

\begin{tabular}{|c|c|c|c|}
\hline $\mathbf{N}^{\mathbf{o}}$ & Alternativas & Frecuencia & Porcentaje \\
\hline a) & $\mathrm{Si}$ & 50 & $13 \%$ \\
\hline b) & No & 195 & $51 \%$ \\
\hline c) & Tal Vez & 138 & $36 \%$ \\
\hline & Total & 383 & $100 \%$ \\
\hline
\end{tabular}

Como se muestra en la tabla 2, se constató que el $51 \%$ de las personas encuestadas expresaron que en la actualidad no tienen la fortaleza y no están preparados ante otro desastre natural, mientras que un $36 \%$ indicaron que tal vez tengan fortaleza y se encuentren preparados ante otro desastre natural, y un $13 \%$ manifestaron que si poseen fortaleza y se encuentren preparados ante otro desastre natural.

Lo que demuestra que una gran parte de las personas encuestadas dieron a conocer que a pesar de las charlas que han recibido por las diferentes instituciones no se sienten preparados ni tienen la fortaleza para soportar otro desastre natural, es muy difícil hacerlo en un evento como el que pasamos el 16 de abril del 2016.

Tabla 3. La resiliencia influye en su desarrollo social

\begin{tabular}{|l|l|l|l|}
\hline $\mathbf{N}^{\mathbf{0}}$ & Alternativas & Frecuencia & Porcentaje \\
\hline a) & Mucho & 247 & $61 \%$ \\
\hline b) & Poco & 120 & $31 \%$ \\
\hline c) & Nada & 16 & $4 \%$ \\
\hline \multicolumn{2}{|l|}{ Total } & 383 & $100 \%$ \\
\hline
\end{tabular}

Se muestra en la tabla 3,65\% de las personas encuestadas indicaron que la resiliencia influye mucho en su desarrollo social, siendo afectado/a por el terremoto mientras que el $31 \%$ manifestaron que la resiliencia influye poco en su desarrollo social, siendo afectado/a por el terremoto y un $4 \%$ expresaron que la resiliencia no influye para nada en su desarrollo social siendo afectado/a por el terremoto. 
Tabla 4. Características que intervienen en la resiliencia

\begin{tabular}{|l|l|l|l|}
\hline $\mathbf{N}^{\circ}$ & Alternativas & Frecuencia & Porcentaje \\
\hline a) & Establecer vínculos con otras personas & 41 & $11 \%$ \\
\hline b) & No considerar que la crisis sea un problema insuperable & 86 & $22 \%$ \\
\hline c) & Aceptar que el cambio forma parte de la vida & 14 & $4 \%$ \\
\hline d) & Actuar con decisión & 75 & $20 \%$ \\
\hline e) & Cultivar la confianza en la capacidad para resolver problemas & 21 & $5 \%$ \\
\hline f) & Poner las cosas en perspectiva & 7 & $2 \%$ \\
\hline g) & Mantener una actitud positiva & 126 & $33 \%$ \\
\hline h) & Cuidarse & 13 & $3 \%$ \\
\hline Total & 383 & $100 \%$ \\
\hline
\end{tabular}

Se observa en la tabla 4,33\% de las personas encuestadas expresaron que mantener una actitud positiva es una de las características que intervienen en la resiliencia para el desarrollo social; mientras que el $22 \%$ manifestaron que no considerar que la crisis sea un problema insuperable es una de las características que intervienen en la resiliencia para el desarrollo social; el 20\% indicaron que actuar con decisión es una de las características que intervienen en la resiliencia para el desarrollo social; el 11\% dijeron que establecer vínculos con otras personas es una de las características que intervienen en la resiliencia para el desarrollo social; el 5\% indicaron que cultivar la confianza en la capacidad para resolver problemas es una de las características que intervienen en la resiliencia para el desarrollo social; el $4 \%$ expresaron que aceptar que el cambio forma parte de la vida es una de las características que intervienen en la resiliencia para el desarrollo social; el 3\% indicaron que cuidarse es una de las características que intervienen en la resiliencia para el desarrollo social; el $2 \%$ indicaron que poner las cosas en perspectiva es una de las características que intervienen en la resiliencia para el desarrollo social. 
Tabla 5: Factores del desarrollo social

\begin{tabular}{|l|l|l|l|}
\hline$N^{\mathbf{0}}$ & Alternativas & Frecuencia & Porcentaje \\
\hline a) & La genética & 236 & $62 \%$ \\
\hline b) & El ambiente social & 147 & $38 \%$ \\
\hline \multicolumn{2}{|l|}{ Total } & 383 & $100 \%$ \\
\hline
\end{tabular}

Según muestra la tabla 5 del total de personas encuestadas, $62 \%$ consideran que la genética es un factor del desarrollo social que ayuda a la resiliencia mientras que el $38 \%$ expresaron que el ambiente social es un factor del desarrollo social que ayuda a la resiliencia.

El $100 \%$ de las personas encuestadas manifestaron que las personas encargadas en ayudar a todas los afectados por el terremoto si emplearon alguna estrategia de resiliencia que ayudo a fortalecer el desarrollo social.

Respecto a la capacidad para sobreponerse ante adversidades incida en su desarrollo social, el haber vivido el terremoto $66 \%$ de las personas encuestadas consideran que tener la capacidad para sobreponerse sí incide en su desarrollo social mientras que el 34 \% indicaron que tener la capacidad para sobreponerse tal vez incide en su desarrollo social al haber vivido el terremoto.

Según Fandiño Rojas D. (2000) la profesión del Trabajo Social en la actualidad y desde sus orígenes como servicio social ha estado presente en la atención de las personas afectadas por desastres naturales o provocados por el hombre. Conforme se ha avanzado en el conocimiento específico acerca de estos, tales como sus fases, aspectos prevenibles, magnitud y otros; así como los efectos del impacto económico, físico, social, psicológico y cultural que tienen en la gente y en el ambiente, el trabajador social ha cumplido y redefinido sus acciones en este campo.

Las áreas de trabajo del trabajador social son: la asistencial, preventivas, promocional-educativa, mediación, transformadora, rehabilitación, planificación, análisis de procesos sociales, necesidades y evaluación, las mismas contribuyen a mejorar el desarrollo e incremento del bienestar social y la calidad de vida de las personas interviniendo de forma crítica y constructiva, 
tratando de potenciar la realización del ser humano incrementando sus potencialidades personales y de promover los recursos comunitarios disponibles y las políticas, (Padilla, 2009).

Por otra parte, se relacionó el tema sobre la atención por parte de un trabajador social, 53\% de las personas encuestadas indicaron que dentro de lo vivenciado si recibieron atención por parte de un trabajador social mientras que el $47 \%$ expresaron no haber recibido atención por el trabajador social.

Del total de entrevistados, $100 \%$ manifestaron que sí deben realizar charlas que ayuden a fomentar la resiliencia y el desarrollo social en las personas que han sido afectadas por el terremoto.

En la casuística se observó, $65 \%$ de las personas encuestadas indicaron que la resiliencia influye mucho en su desarrollo social siendo afectado/a por el terremoto debido a que al poseer la capacidad de sobreponerse ante los diversos obstáculos que se presentan en la vida ayuda a desenvolverse socialmente. Es una realidad que la resiliencia es una reacción interna del ser humano y dependiendo que tan desarrollada la posean les ayudará a desenvolverse mejor socialmente, a su vez deben saber que hay que continuar a pesar del suceso ocurrido.

En febrero 2015, 16 periodistas, en su mayoría de Asia y Latinoamérica, visitaron las zonas afectadas por el gran terremoto del este de Japón y el tsunami. Luego de visitar las zonas afectadas por el gran terremoto de Hanshin-Awaji ocurrido en 1995 en Kansai, el 22 de febrero llegaron a la zona afectada por el Gran Terremoto del Este de Japón de 2011. La actitud de los afectados por el desastre de tratar de ser independientes quedó grabada en la conciencia de los periodistas. (La resiliencia de los sobrevivientes del desastre de Tohoku impacta a periodistas de todo el mundo. 2015).

\section{Conclusiones}

Se pudo conocer dentro de la investigación realizada que la resiliencia sí influye en el desarrollo social de las personas afectadas por el terremoto del 16 de abril del 2016, porque les ayuda a sobreponerse ante las adversidades, les permite ver lo positivo de la vida, a no quedarse 
estancados ante los obstáculos que pudieran presentarse en el suceso, a su vez poder desarrollarse socialmente buscando ayuda y los medios necesarios para comenzar de nuevo.

La intervención del trabajador social contribuyo en la mejora o saber adoptar de forma concreta la resiliencia en las personas afectadas por el terremoto, donde se pudo incrementar el desarrollo social, ayudando a exteriorizar todo aquello que les lastimaba en forma que pudieron tener alivio en ellos mismos, a su vez se realizaron las gestiones necesarias para que las ayudas recibidas lleguen a las personas indicadas.

\section{Referencias bibliográficas}

Elementos para lograr un desarrollo social integral [sitio web]. 2011. Instituto para el Desarrollo Social Integral [consulta 25 agosto 2016]. Disponible en: https://indesi.blogia.com/2011/022801 elementos-para-lograr-un-desarrollo-social-integral.php

FORÉS, A. G. 2008. La resiliencia. Crecer desde la adversidad. Barcelona: Ed. Plataforma actual.

FANDIÑO ROJAS D .2000 Trabajo social e intervención en desastres [consulta 25 agosto 2016]. Disponible en: www.ts.ucr.ac.cr/binarios/docente/pd-000152.pdf

FERNÁNDEZ, A. 2004. Resiliencia y adolescencia. En Resiliencia, ética y prevención, p.43. Buenos Aires: Ed. Gabas.

GROTBERG, E. 2002. Nuevas tendencias en resiliencia. En Resiliencia. Descubriendo las propias fortalezas, p.22 Buenos Aires: Paidós.

GÓMEZ, B. 2010. Resiliencia individual y familiar [consulta 25 agosto 2016]. Disponible en: http://www.avntfevntf.com/imagenes/biblioteca/G\%C3\%B3mez,\%20B.\%20Trab.\%203\%C2\%B A\%20BI\%2009-10.pdf

GARCÍA, U. M., SORIA, S. M. 2010. Factores protectores psicosocioeconómico que determinen resiliencia. p.11 [consulta 25 agosto 2016]. Disponible en: http://repositorio.puce.edu.ec/bitstream/handle/22000/4110/T-PUCE-3388.pdf?sequence=1 
Instituto Nacional de Estadísticas y Censo (INEC) [sitio web]. 2010 [consulta 25 agosto 2016]. Disponible en: http://www.eldiario.ec/noticias-manabi-ecuador/204120-manta-cae-2-puestos-de10-mas-pobladas/

LAGOS P. 2016. Resiliencia desde trabajo social [consulta 25 agosto 2016]. Disponible en:.https://issuu.com/paolalagos6/docs/resiliencia_desde_trabajo_social.do_c24b3e195bbcc9

La resiliencia de los sobrevivientes del desastre de Tohoku impacta a periodistas de todo el mundo [sitio web]. 2015 [consulta 25 agosto 2016]. Disponible en: https://www.jica.go.jp/spanish/news/field/150406_01.html

MELILLO, A., SUÁREZ OJEDA, E. 2002. Resiliencia. Descubriendo las propias fortalezas, p.83. Buenos Aires: Paidós.

MARTÍNEZ, A. 2007. Sociología: Ediciones Maya, p. 24. Madrid

PADILLA, E. 2009. Intervención Social., de [consulta 25 agosto 2016]. Disponible en: http://tscomunitario.blogspot.com/2008/09/trabajo-comunitario-y-trabajo-social.html

SUÁREZ, E. O. 2003. Resiliencia o s capacidad de sobreponerse a la adversidad, 16 p.104. Buenos Aires. Argentina: Medicina y sociedad,

KINARD, E. M. 2008. Cuestiones metodológicas en la evaluación resiliencia en los niños maltratados. Abuso y Negligencia. p.35 Madrid: España. 